\title{
Study on the application of back propagation artificial neural network in thunderstorm forecast
}

\author{
H.F. Wang ${ }^{\mathrm{a}}$, S.H. Li ${ }^{\mathrm{a}}$, Y.B. Hu ${ }^{\mathrm{a}}$, Z.J. Cheng ${ }^{\mathrm{a}}$, W. Li ${ }^{\mathrm{a}}$ and C.Y. Yang ${ }^{\mathrm{a}}$ \\ ${ }^{a}$ Beijing Aviation Meteorological Institute, China \\ Email: wanghongfang@sina.com
}

\begin{abstract}
Thunderstorm is considered as not only one of the top ten ruinous natural disasters by the United Nations, but also the major severe weather of China in summer. Due to its powerful destructive effect and complex forming mechanism, meteorologists have maintained their high level of attentions in thunderstorm forecasting for a long time.Unlike the traditional interpretation of numerical prediction products, artificial neural network model (BPNN) is a nonlinear system simulating the human brain information processing, and it is found that the performance of the BPNN is generally well in characterizing and simulating the typical non-linear structure of the severe weather. In this paper, Based on the NCEP reanalysis field data, forecasting factor are analyzed and selected, and single-site thunderstorm forecasting models based on artificial neural network are established for 20 stations including Beijing. Using mid-term numerical forecast products in June to August of 2008 and 2009 as real-time forecasting input field, the performance of artificial neural network forecasting model are tested, and the results are compared using seven stations' forecasting models and dynamic similar forecasting models to validate the prospects of artificial neural network in forecasting thunderstorms.
\end{abstract}

First, historical data of 1971-2007 are used to statistically analyze the number of thunderstorms occurrences in each month for different site. in most areas of China, thunderstorm probabilities are very low in winter (November to January), less than 1\%, even in February and March in northern region. In summer (JuneAugust) thunderstorms occur more frequently, more than $20 \%$ probabilities. The probability of thunderstorms in the northern reaches its maximum in July, and August for the southern region. In March and October, the probability of thunderstorms dropped suddenly. The climatic probability of occurrence of thunderstorms from north to south is gradually increasing. summer thunderstorms in southern China is not a small probability event.

Second, through the predictor analyzing and selecting using NCEP re-analysis field data in 2000-2007, the single-station thunderstorms forecasting models are built based on artificial neural network for 20 stations. The results of BPNN model are verified using T511 model (a mid-term numerical forecast model) products in summer (June to August) of 2008-2009 as real-time input filed, and compared with the dynamic similarity based model (DSM model).

The results show that nonlinear modeling based artificial neural network is significantly better than dynamic similarity model. The forecast accuracy rate reaches $75 \%$ and $80 \%$, CSI score is 0.411 and 0.502 in 2008 and 2009, respectively. CSI score and the false alarm rate of BPNN model is, respectively, better than that of DSM model. It has a good prospect applying artificial neural network model in the single station thunderstorms forecasting.

Keywords: Artificial neural network; Thunderstorm; Forecasting; Validation 


\section{INTRODUCTION}

Thunderstorm is considered as not only one of the top ten ruinous natural disasters by the United Nations, but also the major severe weather of China in summer. Due to its powerful destructive effect and complex forming mechanism, meteorologists have maintained their high level of attentions in thunderstorm forecasting for a long time, and made a lot of research on the forming mechanism, thunderstorm climatology, convection parameter calculation, statistical forecasting, numerical weather prediction and joint monitoring and nowcasting of satellite and radar (Wagner et al., 2008, Li et al., 1998, Hao et al., 2007, Deng et al., 2007). In recent years, artificial neural networks as a new way, has been gradually applied to the field of weather forecasting and gotten rapid development, which showed a strong capacity in handling of nonlinear problems especially. Because the occurrence of thunderstorms has typical nonlinear characteristics, linear prediction systems based on multivariate statistical regression equations and other traditional interpretation of NWP products cannot solve the problem of non-linearity in the occurrences of thunderstorms, however the multilayer neural network is better able to solve nonlinear problems. During early application of the most common feed-forward network to thunderstorms tentative forecasting it is found that the BP network prediction model based on some significantly associated predictors had good forecasting results (Jing et al., 2005).Since then radiosonde data and lightning location information have been used in the training and testing of neural networks to establish single station thunderstorm forecasting models(Agostino et al., 2005, Zhao et al., 2009), which in turn confirmed the feasibility of forecasting thunderstorms by neural networks.

Based on the NCEP reanalysis field data, forecasting factor are analyzed and selected, and single-site thunderstorm forecasting models based on artificial neural network are established for 20 stations including Beijing. Using mid-term numerical forecast products in June to August of 2008 and 2009 as real-time forecasting input field, the performance of artificial neural network forecasting model are tested, and the results are compared using seven stations' forecasting models and dynamic similar forecasting models to validate the prospects of artificial neural network in forecasting thunderstorms.

\section{DATASETS DESCRIPTION}

Historical data used in this paper for establishing modeling are selected for the period of 2000 to 2007 summer (June to August), a total of 736 days. NCEP analysis field data are used as historical forecasting products, horizontal resolution of $1 \times 1$ degree, four times a day, 00, 06, 12 and 18 UTC respectively, and mid-term numerical forecasting model T511 products are utilized as forecasting fields, horizontal resolution of $1 \times 1$ degree, output products given in 3 hours interval within 72 hours.

Weather phenomenon is extracted from ground routine observation information. It is considered thunderstorm when the weather code is $13,17-19,27,29$ or 89 to 99 . For the matching of the NCEP analysis field and modeling, the in-site observation data are pre-processed, that is: as long as thunderstorm is recorded within 3 hours before or after of $00,06,12$, and 18 Time (UTC), it is considered thunderstorm occurring at the corresponding time.

\section{CLIMATIC CHARACTERISTICS OF THUNDERSTORMS}

In this paper, historical data of 1971-2007 are used to statistically analyze the number of thunderstorms occurrences in each month for different site. A thunderstorm day is defined when there is thunderstorm recorded at any time in that day. And the monthly probabilities of thunderstorms occurring for different site are also calculated respectively.

The calculated result show: in most areas of China except for Zhanjiang, thunderstorm probabilities are very low in winter (November to January), less than 1\%, even in February and March in northern region, Shenyang and Beijing. In summer (June-August) thunderstorms occur more frequently, more than $20 \%$ probabilities (except for Shenyang in August). The probability of thunderstorms in the northern reaches its maximum in July, and August for the southern region. In March and October, the probability of thunderstorms dropped suddenly. The climatic probability of occurrence of thunderstorms from north to south is gradually increasing. Fuzhou, Guangzhou and Zhanjiang are high-incidence area for thunderstorms. More than $10 \%$ of probability in 7 months (March- September) in Fuzhou, especially more than $42 \%$ in July and August; More than $20 \%$ of probability in 6 months (April- September) in Guangzhou, more than $55 \%$ in June and August, near $60 \%$ in August; More than $15 \%$ of probability in 7 months (April- October) in Zhanjiang, more than $50 \%$ in 5 months (May- September ), and in June- August, more than $70 \%$; Thus, summer thunderstorms in southern China is not a small probability event. Thunderstorm days occur mainly during the summer months from June to August, accounted for $60 \%$ or more (except for Zhanjiang), and 
more than $80 \%$ for May- September. Therefore, the study of Chinese summer thunderstorm forecasting is important.

\section{ARTIFICIAL NEURAL NETWORK MODELING}

\subsection{Network structure}

Generally believed to increase the hidden layers can reduce the network errors, improve accuracy, but make the network more complicated, thus increase the network's training time and the emergence of "over-fitting" tendencies. Hornikl et.al proved that network containing one hidden layer can approximate any rational function at any precision. Therefore, in our design of BP network, the three-layer BP network (single hidden layer) is prioritized to consider. However, in practice, for these highly nonlinear thunderstorm weather phenomenon, the use of Layer 3 networks often show a lack of forecasting ability, rather than over-fitting. After a mass of tests, four layers networks (two hidden layers) are selected in the final, the second hidden layer is fixed at a node, and a linear function of the transfer function is set to increase the fitting of nonlinear information.

In the BP network, the choice of hidden layer nodes is very important, not only due to its great impact on the performance of established neural network model, but also the direct cause of over-fitting training. However there is no one scientific and universally applying method for determining the count of hidden nodes. In order to better determine the hidden nodes, in thunderstorm modeling process, a validation dataset is used for establishment of an independent sample return, reducing the number of nodes in hidden layer if fit is good and return is bad, on the contrary, increasing hidden nodes and re-modeling if the fit is not good being lack of forecast ability.

\subsection{Predictors Selecting}

NCEP field parameters (temperature, pressure, humidity, wind) in standard pressure levels are extracted, and the accordingly diagnosis variables associated with the thunderstorms are then calculated. A collection of 53 candidate predictors as a primary group can basically reflects the evolutions of the situation field, temperature and humidity fields, perturbation field, and the circulation field. Variance standardization process of variables is required before the calculation of predictors. As nonlinear characteristics of the artificial neural networks, their choice of predictor is sensitive, additional noise could be easily introduced if the choice of predictors is not appropriate, which in turn cause the system not achieving the training objectives or resulting in over-fitting. Thus the choice of predictors in application must be made site-by-site and predictor-by-predictor.

Table 1. 53 primary predictors of numerical forecast products

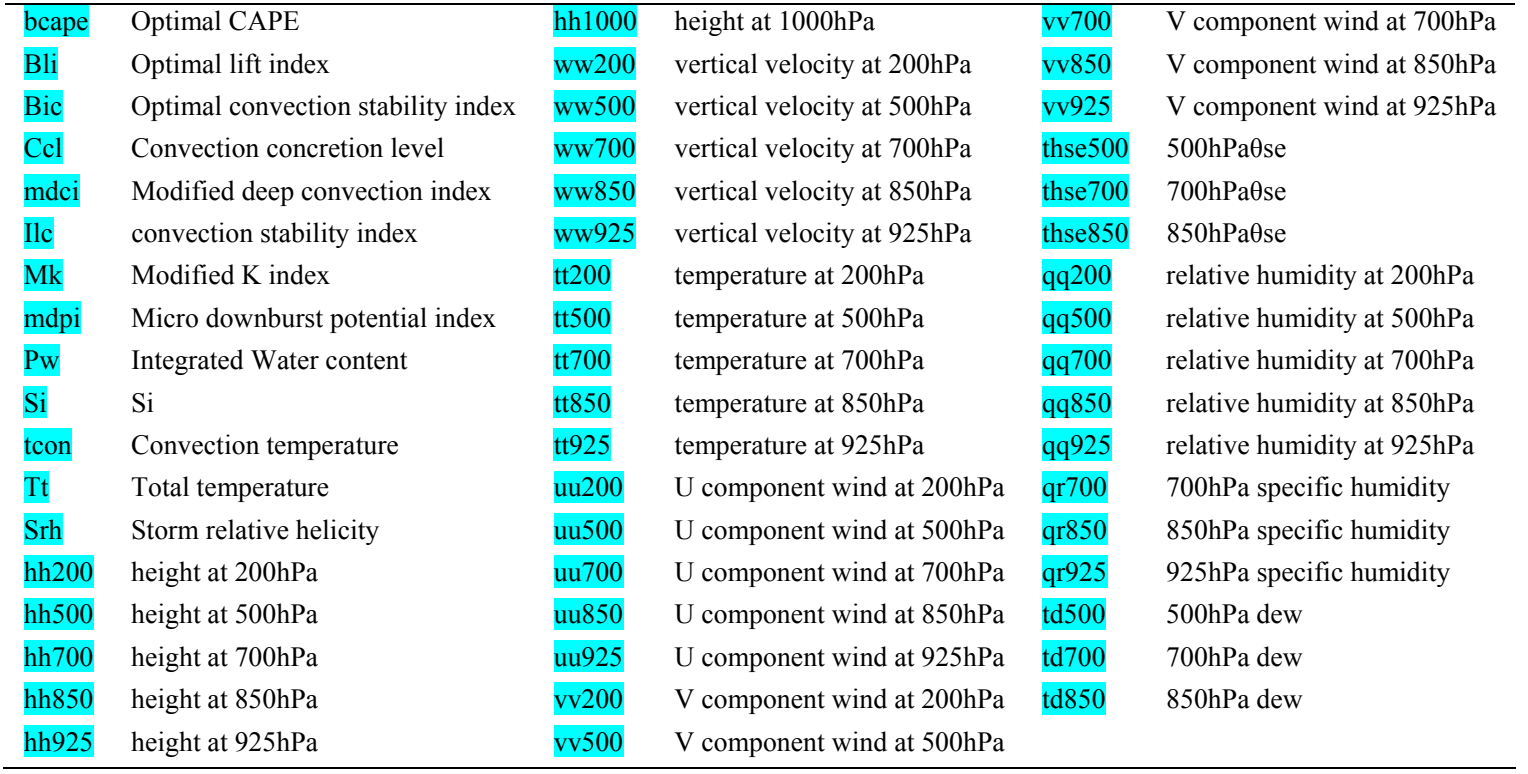

For the forecast station, the correlation between predictors and forecast variables is associated with the weather conditions. How to express this large-scale information into one point plays a key role for single station forecasting. A commonly used method to establish predictor is interpolating gridded predictor field to 
the station. This method can reflect local weather information, but cannot reflect the weather system impacting the station weather, so the stability of the model based on these predictors is not good. In calculating the correlation coefficient, if only considered the vertical and horizontal directions at or around the local site, the relation between true weather situation and forecast elements are unable to address. Therefore, a 'sum-average predictor' method is used in choosing predictors. First, calculate the correlation coefficient between all of the initial predictor fields and thunderstorms, and information including location, related symbols, and whether the situation configuration filed of largest correlation region. Then extract eight grid points where the correlation coefficient are maximum. If the relevant weather situation is a single type, as shown in Figure 1a, and the correlation of the center of big relative humidity value between Beijing station and local station in northwest is obvious, then take the average of the largest eight grid points as 'sumaverage' predictor; If the relevant situation is the configuration field, as shown in Figure $1 \mathrm{~b}$, the field of temperature over Beijing station is symmetric, then take the difference between the averages of 4 maximum and 4 minimum of grid points as the 'sum- average' predictor. The predictors obtained using this 'sumaverage predictor' method may be different for different sites.
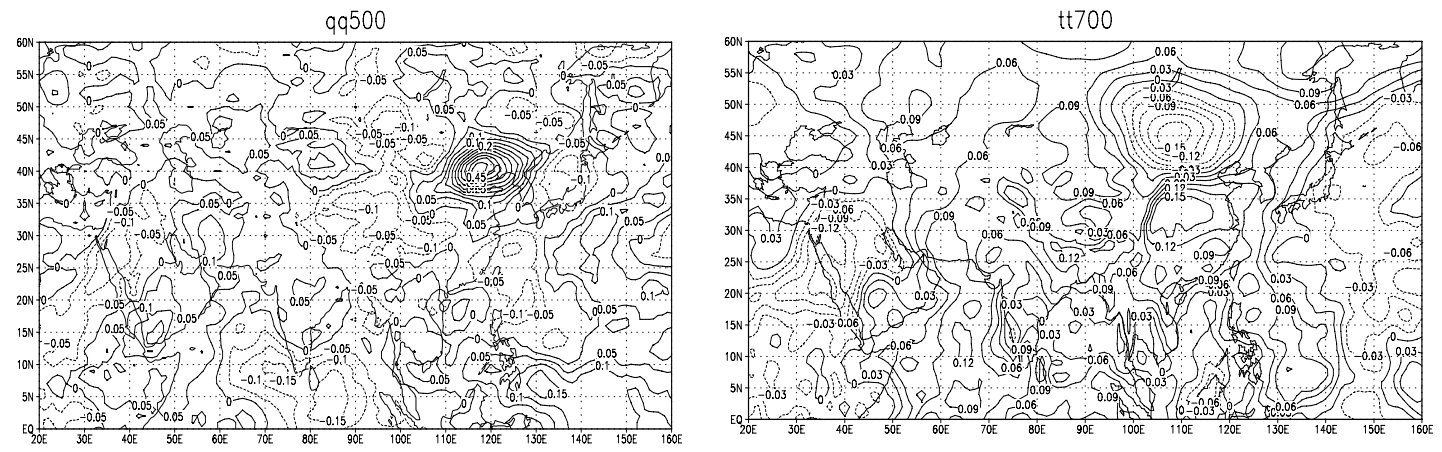

Figure 1. Predictor field configuration distribution

\subsection{Modeling}

The BP neural network provided by MATLAB2009a is selected for modeling. Rational design of BP network model is a complex continuous and sometimes empirical process of adjusting parameters and comparing results. The NCEP analysis fields in summer of 2001-2007 and corresponding in-site observation fields are used as training samples for model training. NCEP analysis fields in summer of 2000 are used as the test samples for the model independent test. The false alarm rate, missing alarm rate and CSI score are used as criteria to evaluate the model. If short of criteria, the network should be re-trained until the criteria are addressed, then the thunderstorms forecast modeling finished.

Because of the small count of thunderstorms samples, the false alarm removing process is to be made before the final prediction outputting. In this paper, a modified $\mathrm{K}$ index method is used in false alarm removing process.

\section{COMPARISON AND ANALYSIS OF FORECAST RESULTS}

\subsection{Test methods}

Thunderstorm forecast models are built based on artificial neural network (BPNN) and dynamic similarity methods (DSM). Using the relevant predictors calculated from T511 mid-term numerical model products forecasting from 12 (UTC) as input filed, the thunderstorm forecasting for the June-August 2009, in the seven regions are made, and the forecast results are examined. CSI index, missing alarm rate $\left(\mathrm{P}_{\mathrm{O})}\right.$ and false alarm rate $\left(\mathrm{F}_{\mathrm{AR}}\right)$ is calculated as follows:

$$
C S I=\frac{N_{\mathrm{A}}}{N_{\mathrm{A}}+N_{\mathrm{B}}+\mathrm{N}_{\mathrm{C}}}, \quad P_{o}=\frac{N_{B}}{N_{A}+N_{B}}, \quad F_{A R}=\frac{N_{C}}{N_{A}+N_{C}}
$$

where, $\mathrm{N}_{\mathrm{A}}$ is the count of correctly forecasting times that thunderstorms are forecasted, and present in fact; $\mathrm{N}_{B}$ is the count of missing alarm times that thunderstorms are not forecasted, but present there; $\mathrm{N}_{C}$ is the count of false alarm times that thunderstorms are forecasted, but absent in fact. 
Wang et al., Study on the application of back propagation artificial neural network in thunderstorm forecast

\subsection{Thunderstorms forecasting BPNN model results test}

Test results are shown in Table 2. Of artificial neural network based thunderstorms forecasting model in summer 2008, the average prediction accuracy rate is 0.749 , missing alarm rate is 0.371 , false alarm rate is 0.455 , CSI score is 0.411 . CSI score 0.565 points up to Zhanjiang, Shantou station as low as 0.29 . In the summer of 2009 the average forecast accuracy is 0.808 , missing alarm rate is 0.296 , false alarm rate is 0.370 , CSI score is 0.502. In 2009 highest CSI score is 0.695 in Zhanjiang station, and CSI score is more than 0.6 in Fuzhou station, the lowest is 0.33 in Guangzhou station. Overall the performance of thunderstorms forecasting BPNN model in 2009 is much better than that in 2008, which indicates that the established thunderstorms forecasting BPNN model has an effective forecasting skill and a relatively high accuracy, so have a good application prospect.

Table 2. Test results of thunderstorms forecasting BPNN model in 2008, 2009 summer (June-August)

\begin{tabular}{|l|l|l|l|l|l|l|l|l|}
\hline \multirow{2}{*}{ Station } & \multicolumn{2}{|l|}{ Summer 2008 } & \multicolumn{2}{l|}{ Summer 2009 } \\
\cline { 2 - 9 } & accuracy & $\begin{array}{l}\text { missing } \\
\text { alarm rate } \\
\text { false alarm }\end{array}$ & $\begin{array}{l}\text { CSI } \\
\text { Score }\end{array}$ & accuracy & $\begin{array}{l}\text { missing } \\
\text { alarm rate }\end{array}$ & $\begin{array}{l}\text { false alarm } \\
\text { rate }\end{array}$ & $\begin{array}{l}\text { CSI } \\
\text { Score }\end{array}$ \\
\hline Shenyang & 0.802 & 0.371 & 0.185 & 0.55 & 0.805 & 0.304 & 0.385 \\
\hline Beijing & 0.824 & 0.385 & 0.579 & 0.333 & 0.828 & 0.35 & 0.485 \\
\hline Nanchang & 0.714 & 0.409 & 0.567 & 0.333 & 0.816 & 0.24 & 0.345 & 0.464 \\
\hline Shantou & 0.758 & 0.55 & 0.55 & 0.29 & 0.828 & 0.357 & 0.526 \\
\hline Fuzhou & 0.681 & 0.333 & 0.381 & 0.473 & 0.816 & 0.297 & 0.161 \\
\hline Guangzhou & 0.758 & 0.312 & 0.607 & 0.333 & 0.77 & 0.375 & 0.583 & 0.375 \\
\hline Zhanjiang & 0.703 & 0.239 & 0.314 & 0.565 & 0.793 & 0.146 & 0.212 \\
\hline $\begin{array}{l}\text { BPNN } \\
\text { Average }\end{array}$ & 0.749 & 0.371 & 0.4547 & 0.411 & 0.808 & 0.2956 & 0.370 \\
\hline DSM & 0.553 & 0.214 & 0.617 & 0.372 & 0.624 & 0.252 & 0.53 \\
\hline
\end{tabular}

\subsection{Results comparison between BPNN model and DSM model}

Dynamic similarity modeling process is composed of two parts: integrate similarity calculation and discrimination. In the discrimination, the 500hpa and $850 \mathrm{hpa}$ height field are used as distinguishing criterion, the similarity coefficient of forecast and history filed is calculated and sorted by descending, and the most similar $\mathrm{N}$ days are selected as discrimination analysis samples. Then calculate convection parameters and related variables in $\mathrm{N}$ days which have the most similar situation filed as primary predictors, based on which the regression model is built, and calculate probability of thunderstorms occurrence within the $\mathrm{N}$ day as a discrimination indicator $\mathrm{YC}$. In the final, the calculated convection parameters and related variables in current day are feeded back to model to get probability of thunderstorms occurrence RC. When $\mathrm{YC} \geq \mathrm{RC}$, no thunderstorm is forecasted, when $\mathrm{YC}<\mathrm{RC}$, thunderstorms are forecasted.

The last two rows of Table 2 show the averages of forecast accuracy, CSI score, false alarm rate, and missing alarm rate of BPNN and DSM model. Fig.2 shows the forecast accuracy, CSI score, false alarm rate, and missing alarm rate both of two models. Comparing the last two rows of Table 2 and Fig. 2 can be seen in the summer of 2008-2009, results of BPNN model are better than DSM model, especially in 2009, the average CSI score of BPNN model is higher than DSM model by 0.138 . For the false alarm rate, BPNN model is better than DSM model, whereas is slightly worse than DSM model for missing alarm rate. Overall, BPNN model is better than DSM model.
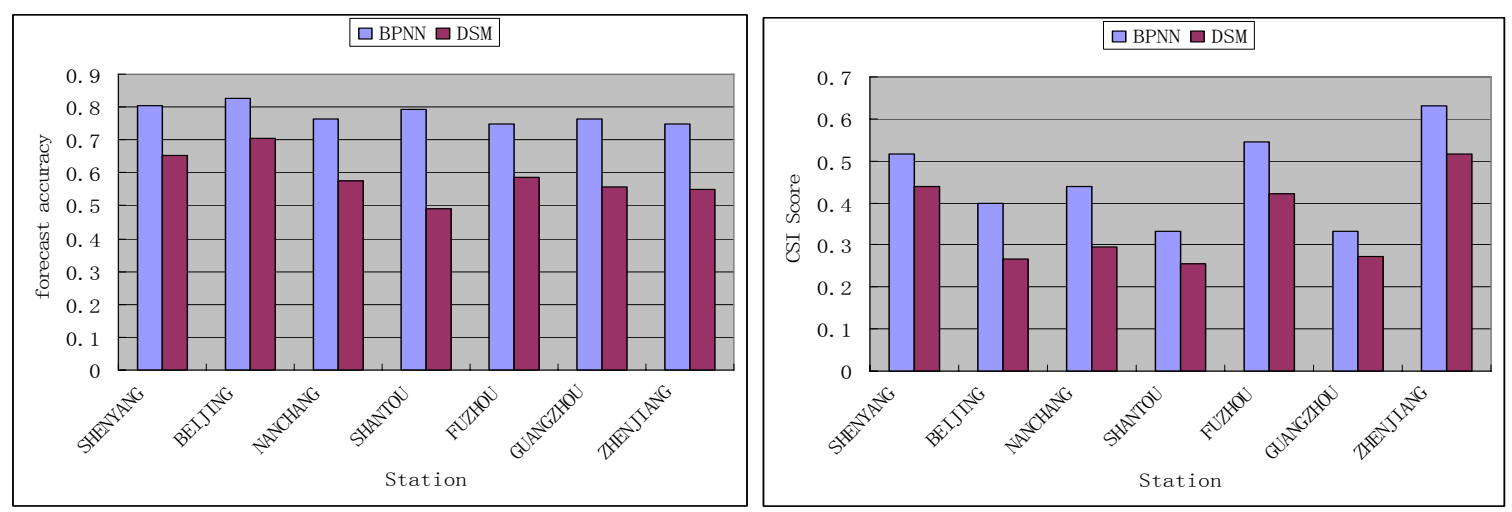

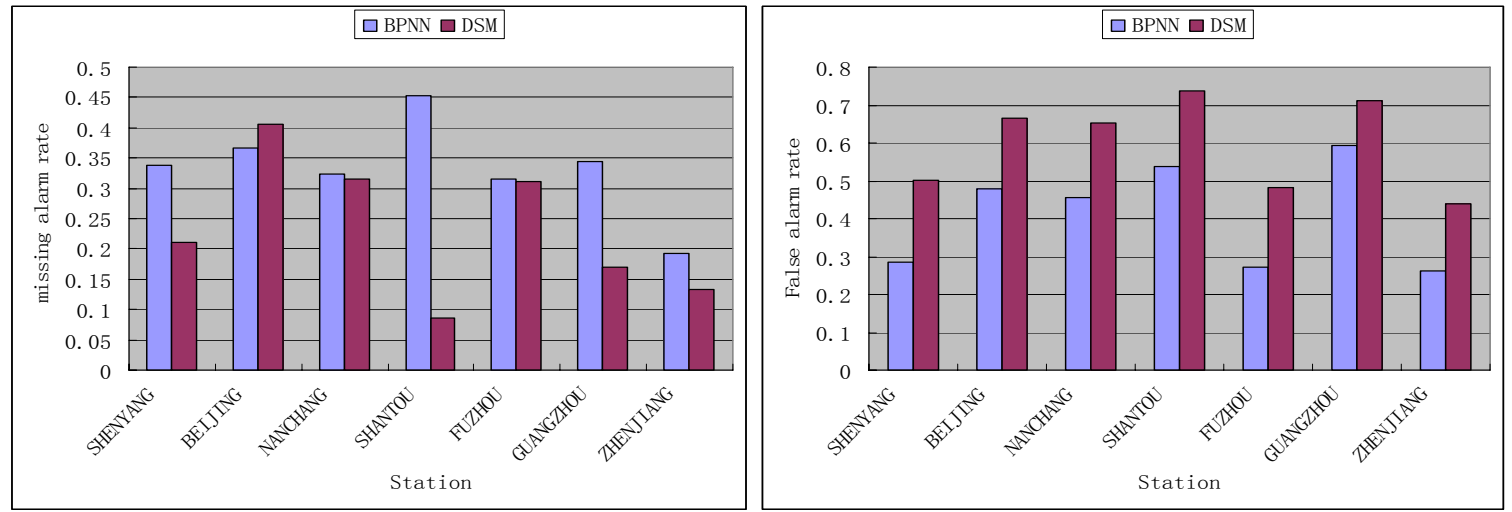

Figure 2. Results comparison of BPNN and DSM model in 2008-2009 (by day)

\section{DISCUSSION AND CONCLUSIONS}

On the consideration of PP method, through the predictor analyzing and selecting using NCEP re-analysis field, the single-station thunderstorms forecasting models are built based on artificial neural network for 20 stations. The results of BPNN model are verified using T511 mid-term numerical forecast products in summer (June to August) of 2008-2009 as real-time input filed, and compared with the DSM model. The results show that the prediction of BPNN model achieves good results, the forecast accuracy rate reaches $75 \%$ and $80 \%$, and CSI score is 0.411 and 0.502 in 2008 and 2009 , respectively.

(1) The correlation between predictors and forecast variables is associated with the weather conditions. Reasonable predictor selection can improve model performance. In this paper, 'sum-average' method is used and the predictors based on it can reflect the spatial and temporal characters of weather system effecting local site.

(2) Dynamic similarity approach modeling needs large enough initial samples to ensure that the selected samples is similar to the intraday weather condition. Nonlinear modeling based artificial neural network is significantly better than dynamic similarity model in the CSI score, forecast accuracy, and the false alarm rate. It has a good prospect that applying artificial neural network model in the single station thunderstorms forecasting.

(3) Result validation shows that the performances of our artificial neural networks are not ideal for certain stations, i.e. high false alarm rate and missing alarm rate, especially in Guangzhou. The probability of thunderstorm occurrence is over $50 \%$ in Guangzhou due to the local convection and oceanic weather character effecting the forecasting of thunderstorm. In the future, local weather character and false alarm removing criterion are focused to improve the false alarm rate of model.

\section{REFERENCES}

Wagner, T.J., W.F. Feltz, and S.A. Ackerman (2008). The Temporal Evolution of Convective Indices in Storm Producing Environments. Weather and Forecasting, 23(5), 786-794.

Li, X.H., and M.Y. Liao (1998). Comprehensive analysis of convective wind in Gunlin at 1997.8.1. Journal of Guangxi Meteorology, 19, 19-20.

Hao, Y., Y.Q. Yao, and Y. Chen (2007). Thunderstorm Potential Trend Forecast Based on Convection Parameters. Meteorological Monthly, 33(1), 51-56.

Deng, B.K. (2007). Suining airport objective prediction of thunderstorms in summer. Journal of Sichuan Meteorology, 27(2), 30-31.

Jing, L. (2005). Neural network forecasting modeling and application. Beijing meteorology press.

Agostino, M. (2005). The use of sounding-derived indices for a Neural Network short-term thunderstorm forecast. Weather and Forecasting, 20(8), 896-917.

Zhao, X., et.al. (2009). A Preliminary Study of Neural Network Based Short-Term Thunderstorm Forecast. Journal of Tropical Meteorology, 25(3), 357-360. 